\title{
Maxillary sinus floor pneumatization and alveolar ridge resorption after tooth loss: a cross-sectional study
}

\author{
Marília Cabral CAVALCANTI ${ }^{(a)}$ \\ Thais Eiler GUIRADO(a) \\ Vitor Marques SAPATA(a) \\ Claudio COSTA ${ }^{(b)}$ \\ Cláudio Mendes PANNUTI (a) \\ Ronald Ernst JUNG(c) \\ João Batista CÉSAR NETO(a) \\ (a) Universidade de São Paulo - USP, School \\ of Dentistry, Division of Periodontology, \\ São Paulo, SP, Brazil. \\ (b) Universidade de São Paulo - USP, School of \\ Dentistry, Division of Radiology, São Paulo, \\ SP, Brazil. \\ (c) University of Zurich, Clinic for Fixed and \\ Removable Prosthodontics and Dental \\ Material Science, Zurich, Switzerland.
}

Declaration of Interests: The authors certify that they have no commercial or associative interest that represents a conflict of interest in connection with the manuscript.

Corresponding Author:

João Batista César Neto

E-mail: jbcesarneto@usp.br

https://doi.org/10.1590/1807-3107BOR-2018.vol32.0064

Submitted: January 11, 2018

Accepted for publication: May 25, 2018

Last revision: June 04, 2018
Abstract: This is a cross-sectional study that aimed to estimate maxillary sinus floor (MSF) pneumatization in single missing tooth of posterior maxilla, by using cone-beam computed tomography (CBCT). CBCT images were analyzed bilaterally and divided into 2 groups: edentulous site (EdS) - edentulous single region of upper second premolar, first or second molars; Tooth site (TS) - contralateral region homologous to the EdS region, with tooth present. Variables evaluated were: sinus height $(\mathrm{SH})$, estimated sinus pneumatization (eSP: $\Delta$ EdS - TS), healed ridge height (HR) and presence of localized sinus pneumatization (LSP) in molars teeth at TS. HR were categorized according to therapeutic option for posterior maxilla. 183 CBCT scans were included and it was observed that EdS presented a higher $\mathrm{SH}$ than the TS $(p<0.001)$ showing an eSP of $0.9 \pm 2.93 \mathrm{~mm}$. First molars presented the highest $\mathrm{SH}$ for both sides, although significant differences were detected when compared to second molars. First molars were mostly affected by LSP at TS (36 out of 43). Individuals with LSP at TS presented lower HR than the ones without LSP ( $p<0.05) .54 \%$ of the cases presenting LSP obtained HR $<5 \mathrm{~mm}$, which indicates sinus lift surgery. The present study showed that tooth loss in posterior maxilla favors sinus pneumatization and the identification of LSP at molar roots seems to indicate a greater necessity for sinus lift surgeries.

Keywords: Maxillary Sinus; Alveolar Process; Dental Implants; Cone-Beam Computed Tomography.

\section{Introduction}

Tooth loss promotes local dimensional changes of hard and soft tissues. ${ }^{1,2,3,4}$ In posterior regions of the upper jaw, maxillary sinus approximation may be present and such event, in combination with bone loss, can difficult implant installation in these sites.

Within the 4 paranasaI sinuses, the maxillary sinuses are the most important for dentistry due to its proximity to teeth. ${ }^{5}$ As the maxillary bone develops, the sinuses cavities are formed and filled by air, a physiological process called pneumatization. ${ }^{6}$ The pneumatization itself causes the maxillary sinuses to expand into the adjacent anatomical structures, being the alveolar process the anatomical region with the highest prevalence rate. ${ }^{5,7}$ It is still a poorly understood process. ${ }^{8}$ There are reports in literature 
of some factors that may influence maxillary sinuses pneumatization, such as heredity, nasal mucous membrane pneumatization, craniofacial configuration, bone density, sinus surgeries, growth hormones, air pressure within the cavity of the sinus and an agerelated process. . $^{7,10,11}$

Some studies have demonstrated that posterior teeth loss can also influence maxillary sinus pneumatization (SP) ${ }^{8,11}$ Radiographically, dental roots appear to penetrate into maxillary sinus floor, however Arx et al. ${ }^{12}$ suggested that pneumatization process makes the sinus floor extends towards the roots. Wehrbein and Diedrich ${ }^{11}$ and Sharan and Madjar ${ }^{8}$ observed in panoramic radiographs a positive correlation between root projection into the maxillary sinus floor and sinus floor pneumatization after tooth extraction. However, panoramic radiographs show superimposition of anatomical structures what may impair the accuracy and reliability of such findings.

Cone-beam computed tomography (CBCT) is currently a complementary diagnostic tool that provide three-dimensional (3D) images. ${ }^{13}$ It significantly reduces the overlapping of anatomical structures and enable a better evaluation of the alveolar bone and the maxillary sinus floor status. ${ }^{14}$ However, there is lack of studies using CBCT scans to analyze sinus floor pneumatization in edentulous posterior maxilla.

Considering the importance of maxillary sinus for implant installation in upper posterior region, an accurate diagnosis and a better understanding of bone remodeling at that particular area may be highly valuable for a precise treatment planning. Thus, the aim of the present study is to investigate, in CBCTs, maxillary sinus height in edentulous areas, estimating the amount of pneumatization. In addition, a secondary analysis will be performed to evaluate the need for maxillary sinus lift and to identify anatomical factors that may favor sinus floor pneumatization.

\section{Methodology}

\section{Study design}

This is a cross-sectional study using cone-beam computed tomography (CBCT) images to assess the maxillary sinus floor pneumatization in edentulous upper jaw regions of second premolars and molars.
This protocol was approved by the Ethical Review Board (Dental School - University of São Paulo) on 5th of August 2016 (ERB approval n. 1.664.769). The study was reported in compliance with STROBE Statement for observational studies.

Initially, 183 CBCT scans were selected from a database of 2574 images of the Department of Stomatology, Division of Radiology (LAPI-FOUSP), School of Dentistry, University of São Paulo (FOUSP), São Paulo, Brazil.

\section{Eligibility criteria}

Inclusion criteria: patients older than 18 years old; CBCT scans showing a single missing tooth in one of the following regions (second premolar or first molar or second molars); presence of the contralateral tooth and presence of a well-defined cortical bone.

Exclusion criteria: presence of upper unerupted tooth; multiple edentulous space; image of recent tooth extraction; presence of bone graft; presence of metallic artifacts that can negatively influence the analysis of the Region of Interest (ROI); presence of cysts, periapical lesions, tumors or traumatic injury at the ROIs and CBCT scans that did not open in the software or did not follow the field of view (FOV) size at the acquisition protocol.

The images were analyzed bilaterally (single edentulous site versus contralateral tooth) and divided into 2 sides:

a. Edentulous Site (EdS): tomographic image of the single edentulous region;

b. Tooth Site (TS): tomographic image of analogous contralateral tooth, correspondent to the edentulous area (second premolar or first molar or second molar).

\section{СВСТ images acquisition and selection}

CBCT scans were obtained using the iCAT Classic ${ }^{\circledR}$ unit (Imaging Sciences, Kavo, USA) with the following acquisition protocol: work scheme of $120 \mathrm{kV}$ acceleration voltage and 3 to $7 \mathrm{~mA}$ beam current; scan time of 20 seconds; flat panel amorphous silico sensor; FOV size of $16 \times 22 \mathrm{~cm} ; 360^{\circ}$ rotation; 14 bits gray scale and voxel size of $0.2 \mathrm{~mm}$.

The XoranCAT® software (Xoran Technologies, USA) was used to review 2574 images in ".xstd" 
extension (Xoran Standard). After that, scans showing a single edentulous region (second premolar, first or second molars) were converted to DICOM extension (Digital Imaging and Communication in Medicine) and exported to be analyzed in Xelis Dental ${ }^{\circledR}$ software (Infinit, South Korea) (Figure 1). The software's licenses belong to LAPI-FOUSP.

\section{Standardization of head and maxillary position prior to evaluation}

Multiplanar (coronal, sagittal and axial) and panoramic reconstructions were used to standardize head and maxillary positions prior to evaluation.

First, in the sagittal reconstruction the adjustment of the patient's head position was done using palatal bone as reference. A line was drawn from the anterior nasal spine (ANS) to the posterior nasal spine (PNS), parallel to the horizontal plane (Figure 2a). In the coronal reconstruction, the nasal cavity floor (NCF) was positioned parallel to the horizontal plane (Figure 2b). After positioning the head, the image of the axial reconstruction was obtained, in which we could observe the cervical of the roots of the upper teeth. From the axial reconstruction, a reference curve was drawn at the midpoint between the buccal and palatal corticals.
Panoramic and transaxial reconstructions were obtained from the reference curve. The ROIs were selected from these reconstructions, always starting at the side where the control tooth is present (TS). The ROIs in the panoramic reconstruction were the central area of the tooth (second premolar, first or second molar) and the central area of the edentulous space.

Transaxial reconstruction was used to perform all measurements in both sides. A line was drawn tangential to the nasal cavity floor until the software's millimeter scale, which was set at the nasal cavity floor position on both sides (Figure 3a - TS; Figure 3b - EdS).

\section{CBCT Evaluation}

The parameters evaluated on CBCTs were:

a. Sinus Height (SH): Linear distance from nasal cavity floor to lowest level of the maxillary sinus floor (Figure 3). This parameter was chosen as primary outcome, and was used as reference to sample size calculation;

b. Estimated Sinus Pneumatization (eSP): difference between sinus height at EdS minus sinus height at TS ( $\triangle \mathrm{EdS}-\mathrm{TS})$;

c. Palatal Crest Height (PC): linear distance from the maxillary sinus floor to lowest point of palatal crest in TS (Figure 3a);

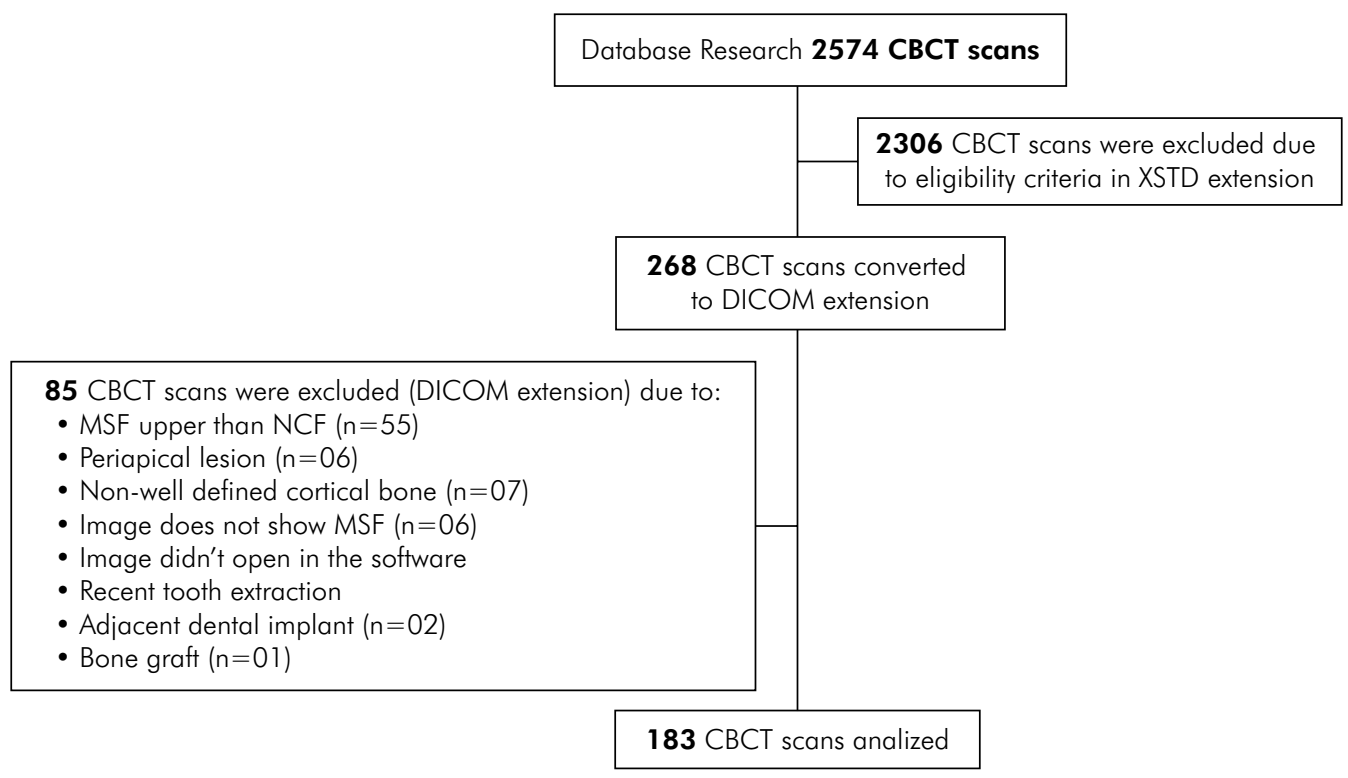

Figure 1. Flowchart: CBCT selection. 

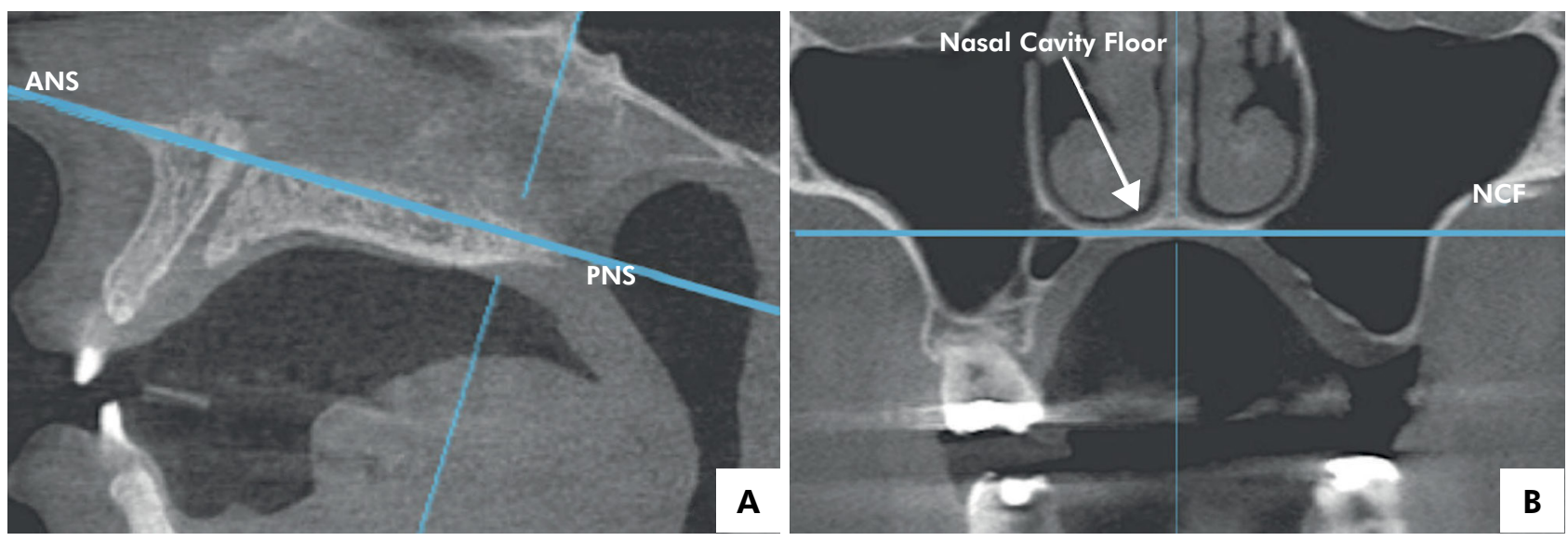

Figure 2. Sagital view of the parallel line to the horizontal plane, from the anterior nasal spine (ANS) to the posterior nasal spine (PNS) (2a). Coronal view of the nasal cavity floor (NCF) positioned parallel to the horizontal plane (2b).

d. Buccal Crest Height (BC): linear distance from the maxillary sinus floor to lowest point of buccal crest in TS (Figure 3a);

e. Healed Ridge Height (HR): linear distance from the maxillary sinus floor to lowest point of alveolar crest in EdS (Figure 3b).

The SH was also separately evaluated according to gender and tooth (second premolar, first or second molar). An additional classification was used to evaluate sinus pneumatization coronal to root apex of molars teeth at TS. The central section was selected in panoramic reconstruction and evaluated at transaxial reconstruction (Figure 3). LSP was considered present when the maxillary sinus floor was coronal to the apex of one of the roots and, absent when the maxillary sinus floor was apical to the level of root apex.

The healed ridge height (HR) was categorized considering therapeutic options for posterior atrophic maxilla proposed by Sanz et al..$^{15}$ Briefly, for "HR < $5 \mathrm{~mm}^{\prime \prime}$ : lateral approach sinus lift is recommended; for "HR between 5 to $8 \mathrm{~mm}$ ": short implants ( $<8 \mathrm{~mm}$ in length) or crestal sinus lift + implants are recommended and; for "HR $>8 \mathrm{~mm}$ " conventional implant placement is recommended.

All measurements were performed by the same, trained and calibrated examiner (MCC). Intra-observer calibration was determined by two measurements of 8 randomly selected CBCT scans with an interval of one week between the first and second evaluation.
An intra-class correlation coefficient of 0.962 was obtained for SH (0.964, CI 95\% 0.831-0.993), HR (0.960, CI 95\% 0.817-0.992), PC (0.940, CI 95\% 0.732-0.988) and BC (0.949, CI 95\% 0.770-0.990).

\section{Sample size calculation}

Sample size calculation was performed with data from a pilot study of $8 \mathrm{CBCT}$ scans and $\mathrm{SH}$ was considered the primary outcome. Considering $1.0 \mathrm{~mm}$ as a clinically relevant difference between TS and EdS, standard deviation of $3.07 \mathrm{~mm}$, alpha $=5 \%$ and $80 \%$ of power, it would be necessary 93 patients to detect the estimated difference by using Paired T-test.
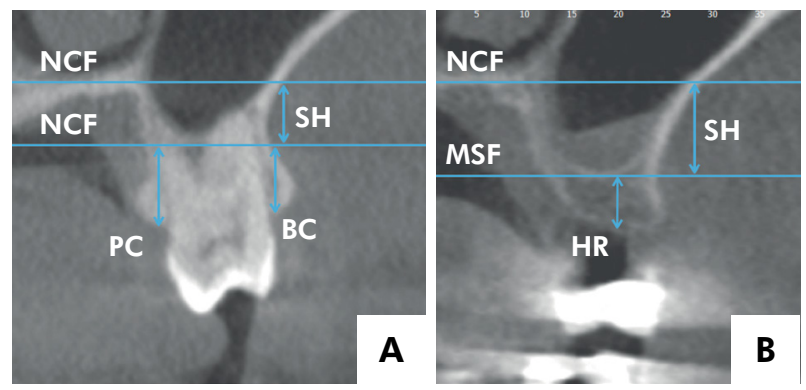

$\mathrm{SH}$ : Linear distance from nasal cavity floor to lowest level of the maxillary sinus floor (MSF) (3a, 3b); PC: linear distance from the maxillary sinus floor to lowest point of palatal crest in TS (3a); BC: linear distance from the maxillary sinus floor to lowest point of buccal crest in TS (3a); HR: linear distance from the maxillary sinus floor to lowest point of alveolar crest in EdS (3b).

Figure 3. Transaxial view. 


\section{Statistical analysis}

Descriptive analysis was performed for each variable of the maxillary sinus and alveolar ridge parameters. The Kolmogorov-Smirnov test was used to verify adherence to the normality curve and the Levene test for homoscedasticity. Since the normal sample distribution and homogeneity of variances criteria were met, parametric tests were used. For the maxillary sinus variables, paired t-test was performed to compare TS and EdS in relation to primary outcome (SH), tooth types and localized sinus pneumatization (LSP). Independent samples t-test was used to compare genders in relation to the variables. One-way Analysis of Variance (ANOVA), followed by a post-hoc test (Tukey) was performed to compare teeth types in relation to $\mathrm{SH}$.

\section{Results}

LAPI-FOUSP database provided 2574 CBCT scans images of which 268 met the main inclusion criteria, i.e. a single superior missing tooth, second premolar, first or second molars, with analogous contralateral tooth present. Eighty-five CBCT scans were subsequently excluded due to reasons reported on Figure 1. Then, 183 were finally selected for analysis (109 female and 74 male patients). The mean age was $47.40 \pm 12.96$ years, ranging from 18 to 80 years. First molars were the most prevalent tooth $(51.92 \%)$, followed by second molars (31.69\%) and second premolars (16.39\%).

\section{Sinus Height (SH)}

The results for $\mathrm{SH}$ are described on Table 1. Overall, EdS presented a higher mean value for $\mathrm{SH}$ $(6.90 \pm 3,15 \mathrm{~mm})$ than TS $(6.00 \pm 3.01 \mathrm{~mm})(\mathrm{p}<0.05)$. The mean difference of the sinus height between EdS and TS (eSP) was $0.90 \pm 2.93 \mathrm{~mm}$. When results were divided according to sex and tooth type, EdS also presented higher mean values than TS ( $p<0.05)$. No differences were observed when males were compared to females for any of the parameters $(\mathrm{p}>0.05)$ (Table 1).

The comparisons of second premolars (SP) vs. first molars (FM) vs. second molars (SM), within each side, showed that tooth type influenced $\mathrm{SH}$. Second molars showed significantly lower mean $\mathrm{SH}$ values at TS (SM: $4.32 \pm 2.49 \mathrm{~mm}$, SP: $5.87 \pm$ $2.57 \mathrm{~mm}, \mathrm{FM}: 7.08 \pm 2.98 \mathrm{~mm}, \mathrm{p}<0.05)$ and $\mathrm{EdS}$ (SM: $5.54 \pm 2.75$ mm, SP: $7.23 \pm 2.93$ mm, FM: $7.63 \pm$

Table 1. Sinus height $(\mathrm{SH})$ measurements $(\mathrm{mm})$ according to overall sample, tooth type, localized sinus pneumatization and sex.

\begin{tabular}{|c|c|c|c|}
\hline Variable & TS & EdS & $\mathrm{eSP}(\triangle \mathrm{EdS}-\mathrm{TS})$ \\
\hline Overall $(n=183)$ & $6,00 \pm 3,01 \mathbf{A}$ & $6,90 \pm 3,15$ B & $0,90 \pm 2,93$ \\
\hline \multicolumn{4}{|l|}{ Sex } \\
\hline Female $(n=109)$ & $6,01 \pm 2,78 \mathbf{A a}$ & $6,90 \pm 3,03 \mathbf{B a}$ & $0,88 \pm 2,78 \mathbf{a}$ \\
\hline Male $(n=74)$ & $6,04 \pm 3,40 \mathbf{A a}$ & $6,98 \pm 3,36 \mathbf{B a}$ & $0,93 \pm 3,13 \mathbf{a}$ \\
\hline \multicolumn{4}{|l|}{ Tooth type } \\
\hline 2 nd premolar $(n=30)$ & $5,87 \pm 2,57 \mathbf{A a}$ & $7,23 \pm 2,93 \mathbf{B a}$ & $1,36 \pm 2,43 \mathbf{a}$ \\
\hline 1 st molar $(n=95)$ & $7,08 \pm 2,98 \mathbf{A a}$ & $7,63 \pm 3,20 \mathbf{B a}$ & $0,55 \pm 3,03 \mathbf{a}$ \\
\hline 2nd molar $(n=58)$ & $4,32 \pm 2,49 \mathbf{A} \mathbf{b}$ & $5.54 \pm 2,75 \mathbf{B b}$ & $1,21 \pm 2,98 \mathbf{a}$ \\
\hline \multicolumn{4}{|c|}{ Localized sinus pneumatization (LSP) } \\
\hline With LSP $(n=43)$ & $8,55 \pm 2,63 \mathbf{A a}$ & $8,28 \pm 3,05 \mathbf{A a}$ & $-0,26 \pm 2,82 \mathbf{a}$ \\
\hline 1 st molar $(n=36)$ & $8,72 \pm 2,60$ & $8,45 \pm 3,26$ & $-0,27 \pm 2,95$ \\
\hline 2nd molar $(\mathrm{n}=7)$ & $7,67 \pm 2,88$ & $7,41 \pm 1,48$ & $-0,25 \pm 2,25$ \\
\hline Without LSP $(n=110)$ & $5,05 \pm 2,70 \mathbf{A b}$ & $6,28 \pm 3,08 \mathbf{B b}$ & $1,22 \pm 2,99 \mathbf{b}$ \\
\hline $1 \mathrm{st}$ molar $(\mathrm{n}=59)$ & $6,07 \pm 2,77$ & $7,14 \pm 3,08$ & $1,06 \pm 2,98$ \\
\hline $2 \mathrm{nd}$ molar $(\mathrm{n}=51)$ & $3,90 \pm 2,09$ & $5,28 \pm 2,82$ & $1,38 \pm 3,04$ \\
\hline
\end{tabular}

Data are described as Mean \pm Standard Deviation in millimeters. Different uppercase letters indicate significant difference between TS and EdS. Different lowercase letters indicate significant difference between tooth type, localized sinus pneumatization and sex. 
$3.20 \mathrm{~mm}, \mathrm{p}<0.05)$. No significant differences were observed between second premolars and first molars. Furthermore, there was no difference between tooth types as regards eSP.

When ROIs were classified and compared according to the presence of localized sinus pneumatization (LSP) at TS, it was observed that only molars present LSP. Within "sites with LSP", no differences for SH were observed between TS and EdS (TS: $8.55 \pm 2.63 \mathrm{~mm}$; EdS: $8.28 \pm 3.05 \mathrm{~mm}, \mathrm{p}>0.05)$. In contrast, in "sites without LSP" there was a significant difference between TS $(5.05 \pm 2.70 \mathrm{~mm})$ and EdS $(6.28 \pm 3.08 \mathrm{~mm})$ for SH (p $<0.05)$. Comparisons within each side demonstrated a similar pattern. At the TS, sites with LSP $(8.55 \pm$ $2.63 \mathrm{~mm}$ ) presented significantly higher $\mathrm{SH}$ mean values when compared to sites without LSP (5.05 \pm $2.70 \mathrm{~mm})(\mathrm{p}<0.05)$. At Eds, sites with LSP $(8.28 \pm$ $3.05 \mathrm{~mm}$ ) presented significantly higher $\mathrm{SH}$ than sites without LSP $(6.28 \pm 3.08 \mathrm{~mm})$. Significant differences were also observed when the estimative of sinus pneumatization (eSP) was compared between "sites with LSP" $(-0.26 \pm 2.82 \mathrm{~mm})$ versus "sites without LSP" $(1.22 \pm 2.99 \mathrm{~mm})(\mathrm{p}=0.002)$ (Table 1$)$.

\section{Palatal crest height (PC), buccal crest height $(B C)$ and healed ridge height (HR)}

The PC had the highest distance from the maxillary sinus floor $(7.59 \pm 5.16 \mathrm{~mm})$ followed by BC $(6.67 \pm$ $2.65 \mathrm{~mm})$ and HR $(5.40 \pm 2.75 \mathrm{~mm})$. According to tooth type, the highest mean value was found for second premolars $(8.16 \pm 2.06 \mathrm{~mm})$ at $\mathrm{PC}$ and the lowest value was for first molars at HR $(5.19 \pm 2.95 \mathrm{~mm})$.

When the height of alveolar ridge was analyzed according to the presence of LSP at molar sites, it was observed that the presence of pneumatization at the dentate site was associated with decreased values of $\mathrm{HR}$ and this difference was statistically significant ( $\mathrm{p}$ $<0.05$ ). On the other hand, no differences for HR were detected when different teeth types were compared ( $p>0.05$ ). See Table 2 for detailed data.

Table 3 shows descriptive statistics and frequency of distribution of HR measurements classified according to treatment option. These were used as a reference for numerical comparisons and inferences during discussion. According to table $3,41 \%$ of the sites presented HR $<5 \mathrm{~mm}(2.82 \pm 1.57 \mathrm{~mm}), 40 \%$ presented HR between $5-8 \mathrm{~mm}(6.20 \pm 0.79 \mathrm{~mm})$ and $19 \%$ presented HR $>8 \mathrm{~mm}(9.42 \pm 1.26 \mathrm{~mm})$. Second premolars presented the lowest frequency of "HR $<5 \mathrm{~mm}$ ", followed by second molars and first molars. In contrast, First Molars showed the highest frequency of "> 8mm of HR, followed by second premolars and second molars. When the sample was divided according to the presence of LSP, LSP sub-group showed $54 \%$ of sites with $\mathrm{HR}<5 \mathrm{~mm}, 37 \%$ with HR between 5-8 $\mathrm{mm}$ and $9 \%$ with HR $>8 \mathrm{~mm}$. The sub-group without LSP presented $38 \%$ of sites with $\mathrm{HR}<5 \mathrm{~mm}, 39 \%$ with HR between 5-8 $\mathrm{mm}$ and $23 \%$ with HR $>8 \mathrm{~mm}$. In summary, the presence of LSF increased the chance of lateral approach sinus lift. In addition, the chance of an implant $>8 \mathrm{~mm}$ decreased when LSP was present.

\section{Discussion}

To the best of authors' knowledge, this is the first study that estimated, through CBCT scans, the maxillary sinus floor pneumatization comparing a single edentulous space versus its contralateral tooth. It was primarily assumed that dental loss could favor sinus pneumatization and, secondarily, that dental region and anatomy of the site could influence this process. The present results confirmed our first hypothesis and showed that, in average, sinus floor was $0.9 \mathrm{~mm}$ coronal at EdS when compared to TS. A surprising result came out from our second question. Before the study, we believed that a pneumatization prior to extraction would favor a

Table 2. Healed Ridge Height (HR) at edentulous sites (EdS) measurements according to tooth type and localized sinus pneumatization.

\begin{tabular}{ll}
\hline$H R$ & \\
\hline 2nd premolar $(n=30)$ & $5,80 \pm 2,90 a$ \\
1st molar $(n=95)$ & $5,19 \pm 2,95 a$ \\
2nd molar $(n=58)$ & $5,52 \pm 2,33 a$ \\
Localized sinus pneumatization & \\
With LSP $(n=43)$ & $4,28 \pm 2,45 a$ \\
Without LSP $(n=110)$ & $5,72 \pm 2,73 \mathrm{~b}$ \\
\hline
\end{tabular}

Data are described as Mean \pm Standard Deviation in millimeters. Different lowercase letters indicate significant difference between tooth type and localized sinus pneumatization. 
Table 3. Healed Ridge Height (HR) classified as $<5 \mathrm{~mm}, 5-8 \mathrm{~mm}$ and $>8 \mathrm{~mm}$ according to tooth type and localized sinus pneumatization.

\begin{tabular}{|c|c|c|c|}
\hline Variable & $<5 \mathrm{~mm}$ & $5-8 \mathrm{~mm}$ & $>8 \mathrm{~mm}$ \\
\hline \multicolumn{4}{|l|}{ Tooth type } \\
\hline 2nd premolar $(n=30)$ & $2,93 \pm 1,65 /(37 \%)$ & $6,52 \pm 0,80 /(47 \%)$ & $10,14 \pm 2,06 /(16 \%)$ \\
\hline 1st molar ( $\mathrm{n}=95)$ & $2,55 \pm 1,55 /(44 \%)$ & $5,97 \pm 0,72 /(35 \%)$ & $9,45 \pm 1,21 /(21 \%)$ \\
\hline 2nd molar $(n=58)$ & $3,27 \pm 1,54 /(40 \%)$ & $6,33 \pm 0,82 /(45 \%)$ & $8,96 \pm 0,63 /(15 \%)$ \\
\hline Overall \pm SD / \% & $2,82 \pm 1,57 /(41 \%)$ & $6,20 \pm 0,79 /(40 \%)$ & $9,42 \pm 1,26 /(19 \%)$ \\
\hline \multicolumn{4}{|c|}{ Localized sinus pneumatization } \\
\hline \multicolumn{4}{|l|}{ With LSP } \\
\hline 1 st molar $(n=36)$ & $2,30 \pm 1,58 /(53 \%)$ & $5,74 \pm 0,54 /(36 \%)$ & $8,90 \pm 0,65 /(11 \%)$ \\
\hline 2nd molar $(n=07)$ & $3,25 \pm 1,03 /(57 \%)$ & $5,80 \pm 0,88 /(43 \%)$ & - \\
\hline Overall \pm SD / \% & $2,46 \pm 1,53 /(54 \%)$ & $5,75 \pm 0,60 /(37 \%)$ & $8,90 \pm 0,65 /(09 \%)$ \\
\hline \multicolumn{4}{|l|}{ Without LSP } \\
\hline 1st molar $(n=59)$ & $2,76 \pm 1,53 /(39 \%)$ & $6,12 \pm 0,80 /(34 \%)$ & $9,59 \pm 1,30 /(27 \%)$ \\
\hline 2nd molar $(n=51)$ & $3,28 \pm 1,64 /(37 \%)$ & $6,39 \pm 0,81 /(45 \%)$ & $8,86 \pm 0,62 /(18 \%)$ \\
\hline Overall \pm SD / \% & $3,00 \pm 1,58 /(38 \%)$ & $6,17 \pm 0,80 /(39 \%)$ & $9,37 \pm 1,13 /(23 \%)$ \\
\hline
\end{tabular}

Mean \pm Standard deviation / (Percentage).

future pneumatization after extraction. However, our data showed the opposite, the coronal position of sinus floor doesn't change in regions of previous sinus pneumatization. And, the changes mostly happen at the regions without previous pneumatization. Based on such findings, we may suggest to the practitioner whether a pneumatization seems to occur in the periapical radiograph, the site is candidate for a CBCT examination prior to extraction. It can improve the precision of treatment planning and provide a more realistic scenario in terms of complexity. For instance, a grafting procedure may be strategically included in the extraction procedure with the objective of decreasing the chance of a sinus graft and, hence, decreasing the complexity and invasiveness of the treatment.

Evaluating each tooth type separately, the present results demonstrated that sinus pneumatization occurs with greater intensity in the second premolars and first molars. On the other hand, the evaluation of healed ridges (HR) showed that first molars presented the lowest HR values. This controversy may be explained by the high incidence of LSP in first molars (36 out of $95 \mathrm{FM}$ ) resulting in a decrease ridge height, less prone to receive an implantsupported rehabilitation. This finding seemed to be in line with our hypothesis that LSP prior to extraction would be a factor that could favor sinus pneumatization after extraction. However, the absence of differences between TS and EdS within LSP sub-group contradicts such expectation and reinforces the hypothesis that previous studies using $2 \mathrm{D}$ radiographs ${ }^{8}$ may have underestimated the presence of sinus pneumatization prior to extractions.

According to the present data it seems that if a LSP is present at the moment of the extraction, just a slight change will occur after healing, because in that particular site the coronal migration of sinus floor had already occurred. Two additional findings of the present study corroborate with this hypothesis. First, the abrupt difference of eSP observed when individuals with and without LSP were compared $(-0.26 \pm 2.82$ and $1.22 \pm 2.99$ for each sub-group respectivelly, $p<0.05)$. Second, the difference for HR comparing individuals with and without LSP $(4.28 \pm 2.45$ and $5.72 \pm 2.73$ for each sub-group respectively, $\mathrm{p}<0.05)$. These findings suggest that sinus pneumatization prior to extraction doesn't predispose to a more pronounced migration of sinus floor towards the ridge, but may result in a decreased HR.

In clinical standpoint, the present findings strongly suggest that the need for lateral window sinus lift approach could be more precisely estimated if $\mathrm{CBCT}$ evaluation is performed prior to extraction, 
which is not the case for conventional radiographs. ${ }^{8}$ The present study also observed no differences between the genders for the pneumatization of the maxillary sinus floor, which is in agreement with the previous studies that did not find differences in sinus volume after third decade of $l i f e^{16}$ and in sinus depth between genders. ${ }^{17}$

Sharan and ${ }^{8}$ in a retrospective study, used linear measurements to evaluate maxillary sinus floor changes, however, they used panoramic radiographs and different methodology. They compared, at the same site, mean values of sinus pneumatization before and at least 6 months after tooth extraction, and found a mean difference of $1.83 \pm 2.46 \mathrm{~mm}$ between each time point. A similar figure was observed when they compared the healed edentulous site with their contralateral teeth $(2.18 \pm 2.89 \mathrm{~mm})$. Comparing the present eSP $(0.90 \pm$ $2.93 \mathrm{~mm}$ ) with the mean value of pneumatization found by Sharan and Madjar, ${ }^{8}$ it seems that they found a pneumatization 2 times more severe. The magnitude of such difference may be credited to the imaging technique and for the inclusion of multiple and single sites by Sharan and Madjar. ${ }^{8}$ However, they observed only $0.54 \pm 1.70 \mathrm{~mm}$ of pneumatization for single tooth extractions, which is close to our findings. Similar findings (1.30 \pm $0.27 \mathrm{~mm}$ of estimated pneumatization) were observed in a recent study by Levi et al. ${ }^{18}$ that compared pre and post extraction panoramic radiographs from a database. The authors also observed a less intense pneumatization in the group that was treated with socket preservation $(0.30 \pm 0.10 \mathrm{~mm})$, what is in agreement with a pilot study by Rasperini et al. ${ }^{19}$

Vertical bone loss at posterior maxilla is a combination of SP and alveolar crest resorption. When the mean difference $(2.2 \mathrm{~mm}+0.9 \mathrm{~mm})$ between palatal crest height $(7.59 \pm 5.16 \mathrm{~mm})$ and healed ridge height $(5.40 \pm 2.75 \mathrm{~mm})$ is added to the mean eSP $(0.90 \pm 2.93 \mathrm{~mm})$, approximately $3.12 \mathrm{~mm}$ of posterior maxilla height loss can be expected. Based on the present findings it can be estimated that, at maxillary posterior sites, sinus pneumatization corresponds to about $30 \%$ of height loss and socket remodeling corresponds to about $70 \%$ of this process.
Nunes et al. ${ }^{20}$ also performed a retrospective anatomical study using CBCT images in upper posterior region but, they included multiple edentulous spaces (from first premolars to second molars). Moreover, they evaluated the need for sinus augmentation procedure (taking into account the dimensions of the alveolar ridge), not measurements of sinus floor position. Similarly, they also considered $\mathrm{HR}<5 \mathrm{~mm}$ an indication for lateral window sinus lift approach and implant installation in a second stage. Despite the methodological differences, $41 \%$ of Nunes et al. ${ }^{20}$ sample showed less than $5 \mathrm{~mm}$ of ridge height, result that is similar to ours. Both studies found that first molar was the region with the highest chance of sinus lift need, but slightly different percentages were observed.

The identification of LSP prior to extraction seems to be effective on predicting the need for a future lateral window sinus lift. There is still some controversy what is the ideal approach when the HR ranges from 5 to $8 \mathrm{~mm}$. According to Sanz et al., ${ }^{15}$ the installation of short implants is a valid therapeutic option to avoid invasive surgeries of maxillary sinus augmentation. Also, Thoma et al. ${ }^{21}$ described that surgeries for sinus lift has higher complication rate, increased morbidity, costs and longer surgical time. Thus, shorter dental implants should be the preferred treatment alternative, with a high survival rate, in atrophic jaws. Most of the authors agree that lateral window approach should be indicated in $\mathrm{HR}<5 \mathrm{~mm}^{20,22}$ and that implant placement in HR> $>\mathrm{mm}$ is a safe and predictable procedure. In this sense, the present data allows us to infer that, the presence of LSF increased the chance of lateral window sinus lift in about $42 \%$ (38\% versus $54 \%$, for without and with LSP, respectively). In addition, the chance of an implant $>8 \mathrm{~mm}$ increased $150 \%$ when LSP was absent $(23 \%$ versus $9 \%$, for without and with LSP, respectively).

Despite such interesting results, the present study has some important limitations that should be taken into consideration. Due to the retrospective design, there is no information about tooth loss cause, extent of surgical trauma during extraction, amount of time without the tooth, the position of the maxillary sinus floor and its relationship with the root apex 
immediately before tooth extraction. Although a study by Hamdy and Abdel-Wahed ${ }^{14}$ demonstrated a considerable maxillary sinus volume symmetry between the right and left side of the maxillae, it is not possible to assume that the same had happened in our sample. More prospective clinical studies associated with CBCT analyses are necessary to confirm the present findings, to clarify these possible limitations and to better understand the surgical needs in posterior maxilla.

\section{References}

1. Araújo MG, Lindhe J. Dimensional ridge alterations following tooth extraction. An experimental study in the dog. J Clin Periodontol. 2005 Feb;32(2):212-8. https://doi.org/10.1111/j.1600-051X.2005.00642.x

2. Benic $\mathrm{Gl}$, Hämmerle $\mathrm{CH}$. Horizontal bone augmentation by means of guided bone regeneration. Periodontol 2000. 2014 Oct;66(1):13-40. https://doi.org/10.1111/prd.12039

3. Araújo MG, Silva CO, Misawa M, Sukekava F. Alveolar socket healing: what can we learn? Periodontol 2000. 2015 Jun;68(1):122-34. https://doi.org/10.1111/prd.12082

4. Misawa M, Lindhe J, Araúio MG. The alveolar process following single-tooth extraction: a study of maxillary incisor and premolar sites in man. Clin Oral Implants Res. 2016 Jul;27(7):884-9. https://doi.org/10.1111/clr.12710

5. Lana JP, Carneiro PM, Machado VC, Souza PE, Manzi FR, Horta MC. Anatomic variations and lesions of the maxillary sinus detected in cone beam computed tomography for dental implants. Clin Oral Implants Res. 2012 Dec;23(12):1398-403. https://doi.org/10.1111/j.1600-0501.2011.02321.x

6. Del Fabro M, Testori T. Maxillary sinus surgery and alternatives in treatment: Anatomy of the maxillary sinus. Berlin: Quintessence; 2009.

7. Sánchez-Pérez A, Boracchia AC, López-Jornet P, BoixGarcía P. Characterization of the Maxillary Sinus Using Cone Beam Computed Tomography. A Retrospective Radiographic Study. Implant Dent. 2016 Dec;25(6):762-9. https://doi.org/10.1097/ID.0000000000000485

8. Sharan A, Madjar D. Maxillary sinus pneumatization following extractions: a radiographic study. Int J Oral Maxillofac Implants. 2008 Jan-Feb;23(1):48-56.

9. Shapiro R, Schorr S. A consideration of the systemic factors that influence frontal sinus pneumatization. Invest Radiol. 1980 May-Jun;15(3):191-202. https://doi.org/10.1097/00004424-198005000-00004

10. Thomas A, Raman R. A comparative study of the pneumatization of the mastoid air cells and the frontal and maxillary sinuses. AJNR Am J Neuroradiol. 1989 Sep-Oct;10(5 Suppl):S88.

\section{Conclusions}

The present study showed that tooth loss in posterior maxilla favors the maxillary sinus pneumatization. Moreover, the maxillary sinus pneumatization may vary according to tooth type and region. And the presence of localized sinus pneumatization in molars seems to indicate a greater change of lateral window sinus lift approach and a decreased healed ridge height.

11. Wehrbein H, Diedrich P. [Progressive pneumatization of the basal maxillary sinus after extraction and space closure]. Fortschr Kieferorthop. 1992 Apr;53(2):77-83. German. https://doi.org/10.1007/BF02164641

12. Arx T, Fodich I, Bornstein MM. Proximity of premolar roots to maxillary sinus: a radiographic survey using cone-beam computed tomography. J Endod. 2014 Oct;40(10):1541-8. https://doi.org/10.1016/i.joen.2014.06.022

13. Abramovitch K, Rice DD. Basic principles of cone beam computed tomography. Dent Clin North Am. 2014 Jul;58(3):463-84. https://doi.org/10.1016/i.cden.2014.03.002

14. Hamdy RM, Abdel-Wahed N. Three-dimensional linear and volumetric analysis of maxillary sinus pneumatization. J Adv Res. 2014 May;5(3):387-95. https://doi.org/10.1016/i.jare.2013.06.006

15. Sanz M, Donos N, Alcoforado G, Balmer M, Gurzawska $K$, Mardas N, et al. Therapeutic concepts and methods for improving dental implant outcomes. Summary and consensus statements. The 4th EAO Consensus Conference 2015. Clin Oral Implants Res. 2015 Sep;26 Suppl 11:202-6. https://doi.org/10.1111/clr.12674

16. Jun BC, Song SW, Park CS, Lee DH, Cho KJ, Cho JH. The analysis of maxillary sinus aeration according to aging process; volume assessment by 3 -dimensional reconstruction by high-resolutional CT scanning. Otolaryngol Head Neck Surg. 2005 Mar;132(3):429-34. https://doi.org/10.1016/i.otohns.2004.11.012

17. Wagner F, Dvorak G, Nemec S, Pietschmann P, Figl M, Seemann R. A principal components analysis: how pneumatization and edentulism contribute to maxillary atrophy. Oral Dis. 2017 Jan;23(1):55-61. https://doi.org/10.1111/odi.12571

18. Levi I, Halperin-Sternfeld M, Horwitz J, Zigdon-Giladi H, Machtei EE. Dimensional changes of the maxillary sinus following tooth extraction in the posterior maxilla with and without socket preservation. Clin Implant Dent Relat Res. 2017 Oct;19(5):952-8. https://doi.org/10.1111/cid.12521 
- Cavalcanti MC, Guirado TE, Sapata VM, Costa C, Pannuti CM, Jung RE et al.

19. Rasperini G, Canullo L, Dellavia C, Pellegrini G, Simion M. Socket grafting in the posterior maxilla reduces the need for sinus augmentation. Int J Periodontics Restorative Dent. 2010 Jun;30(3):265-73.

20. Nunes LS, Bornstein MM, Sendi P, Buser D. Anatomical characteristics and dimensions of edentulous sites in the posterior maxillae of patients referred for implant therapy. Int J Periodontics Restorative Dent. 2013 MayJun;33(3):337-45. https://doi.org/10.11607/prd.1475
21. Thoma DS, Zeltner M, Hüsler J, Hämmerle CH, Jung RE. EAO Supplement Working Group 4 - EAO CC 2015 Short implants versus sinus lifting with longer implants to restore the posterior maxilla: a systematic review. Clin Oral Implants Res. 2015 Sep;26 Suppl 11:154-69. https://doi.org/10.1111/clr.12615

22. Al-Dajani M. Recent trends in sinus lift surgery and their clinical implications. Clin Implant Dent Relat Res. 2016 Feb;18(1):204-12. https://doi.org/10.1111/cid.12275 